\title{
Classification of small UAVs and birds by micro-Doppler signatures
}

\author{
P. Molchanov*, K. Egiazarian*, J. Astola*, R. I. A. Harmanny ${ }^{\dagger}$, J. J. M. de Wit ${ }^{\ddagger}$ \\ *Department of Signal Processing, Tampere University of Technology, Tampere, Finland \\ firstname.lastname@tut.fi \\ ${ }^{\dagger}$ Thales Nederland B.V., Delft, The Netherlands \\ ronny.harmanny@nl.thalesgroup.com \\ $\ddagger$ Department of Radar Technology, TNO, The Hague, The Netherlands \\ jacco.dewit@tno.nl
}

\begin{abstract}
The problem of unmanned aerial vehicles classification using continuous wave radar is considered in this paper. Classification features are extracted from micro-Doppler signature. Before the classification, the micro-Doppler signature is filtered and aligned to compensate the Doppler shift caused by the target's body motion. Eigenpairs extracted from the correlation matrix of the signature are used as informative features for classification. The proposed approach is verified on real radar measurements collected with $9.5 \mathrm{GHz}$ radar. Planes, quadrocopter, helicopters and stationary rotors as well as birds are considered for classification. Moreover, a possibility of distinguishing different number of rotors is considered. The obtained results show the effectiveness of the proposed approach. It provides capability of correct classification with a probability of around $95 \%$.
\end{abstract}

\section{INTRODUCTION}

Unmanned Aerial Vehicles (UAVs) are a special type of aerial targets which have small physical size as they are pilotless. Classification of them by High Range Resolution Radar Profiles (HRRPs) is problematic because sub centimetre resolution is required to capture a spatial structure of targets less than $100 \mathrm{~cm}$ in length.

The contributions of rotating turbine or propeller blades in radar backscattering in the form of micro-Doppler contents [1] can contain additional classification information about UAV characteristics. These micro-Doppler contributions are unique for different types of UAVs [2].

Exploiting micro-Doppler signatures for classification allows the use of low-cost continuous wave radar in automatic target recognition (ATR) systems. The main contribution of this paper is in the definition and extraction of new robust features that can be extracted from the micro-Doppler signature. The proposed features are based on basis functions of the micro-Doppler signature and they can be extracted by applying Eigen or Singular Value Decomposition. The resulting features are orthogonal and unique providing essential and uncorrelated information about the target under consideration.

Previously, the main attention of researches was paid to target classification by micro-Doppler signatures of humans, animals or big aerial targets [3]. The UAV classification based on micro-Doppler signature is a novel topic.

In this paper we consider in detail all processing steps required for feature extraction after a target has been detected. These steps include estimation and filtering of the microDoppler signature, alignment of the micro-Doppler signature in order to compensate the Doppler contribution of the target's body motion and the actual feature extraction. We assess the robustness of the extracted features by feeding them to different types of classifiers. The assessment is performed using measured radar data of different types of targets such as aircraft, a quadrocopter, helicopters, stationary rotors and birds. Each target type induces a unique micro-Doppler signature depending on the velocity, orientation and direction of the motion.

The rest of the paper is organized as follows. The proposed approach is introduced in details in the Section II. Then experimental verification is given in the Section III. Finally, conclusions are provided.

\section{PROPOSED APPROACH}

\section{A. General scheme}

The general scheme of the proposed ATR system consists of the following steps:

a) Micro-Doppler signature estimation: To obtain microDoppler signature, the received signal must be transformed to time-frequency (TF) domain. The simplest and effective way is to apply Short-Time Frequency Transform (STFT).

b) Filtering: The radar and the analogue-to-digital converter (ADC) cause time-independent interference (noise pattern) to the true signal. This noise can be estimated by processing the signal-free record. When the noise pattern is estimated it can be removed from the actual radar records. Good robustness is shown by the "spectral subtraction" [4] method and it is implemented in this work.

c) Alignment of the micro-Doppler signature: We want to classify targets by micro-Doppler signature, meaning by contribution from propellers and not from the target' body. The motion of the body must be estimated and compensated before extracting features from micro-Doppler signature.

d) Feature extraction: The main contribution of the proposed ATR scheme is in extracted features from TF domain. The distinguishing features will be extracted in the form of basis functions of the spectrogram which are processed by amplitude of Fourier transform.

e) Classifier: In this paper, three different types of classifiers are considered in order to evaluate the robustness of the proposed features. The first two classifiers are the linear and non-linear Support Vector Machines (SVMs) belonging to non-probabilistic classifiers, and the third one is the Naive 
Bayes Classifier (NBC) belonging to probabilistic linear classifiers.

\section{B. Estimation of micro-Doppler signature}

Assume $s \in \mathbb{C}^{N}$ is a received Doppler signal in the radar, where $N$ is the length of the signal in number of temporal samples. The received Doppler signal is obtained by I/Q demodulation of the reflected radio wave. In the digital form it can be written as:

$$
s(n)=\sum_{k=1}^{K} a_{k} e^{j f_{k}(n)}+\epsilon(n),
$$

where $j=\sqrt{-1}$ is imaginary unit, $K$ is the number of scatters, $a_{k}$ is reflectivity of the $k$-th scatterer, $f_{k}(n)$ is Doppler frequency shift of the $k$-th scatterer, $\epsilon(n)$ is additive noise.

The micro-Doppler signature of the moving target is estimated as the magnitude of the spectrogram of the Doppler signal:

$S(f, t)=\left|\sum_{m=0}^{M-1} w(m) s(m+(t-1)(N-L)) e^{-j 2 \pi f m / M}\right|$,

where $w(m)$ is the smoothing window function of length $M$, for instance, Hamming window can be exploited; $f$ is the frequency index; $L$ is the overlap of successive Fourier lengths, expressed in samples ; the dimensionality of the spectrogram is $S \in \mathbb{R}^{M \times Q}, Q=\frac{N-L}{M-L}$.

\section{Filtering}

The noise $\epsilon$ could be removed from the observation $s$ by the "spectral subtraction" noise reduction procedure. The main idea of this approach is subtraction of an estimate of the average noise spectrum from the noisy signal spectrum. We assume that noise exhibits a static frequency profile with varied gain. Spectral subtraction is applied in our paper to reduce radar interference effects.

The average noise spectrum is estimated by periodogram:

$$
\Upsilon(f)=\frac{1}{Q} \sum_{t=1}^{Q} S^{(\epsilon)}(f, t),
$$

where $S^{(\epsilon)}$ is a STFT of noise only signal $\epsilon$.

The gain of noise is estimated as a normalized length of the projection of the noisy signal spectrum onto noise spectrum:

$$
G(t)=\sum_{f=1}^{M} \frac{\Upsilon(f) S(f, t)}{\|\Upsilon\|^{2}}
$$

where $\|\Upsilon\|=\sqrt{\Upsilon(1)^{2}+\Upsilon(2)^{2}+\ldots+\Upsilon(M)^{2}}$ is a Euclidean norm of the noise pattern.

The noise can be removed from the signature as:

$$
U(f, t)=S(f, t)-G(t) \Upsilon(f) .
$$

\section{Alignment of the micro-Doppler signatures}

In order to extract behaviour of micro-Doppler features the motion of the target must be compensated. This can be done by tracking the change of velocity of the target's body.

Let us assume that target's body is a scatterer with the highest reflectivity coefficient. Therefore, the target's body will appear as the maximum at the STFT. We need to track this maximum and then compensate the motion. However, the contribution of the stationary clutter is not removed yet. For this purpose we propose to apply a weighting function before estimating the maximum:

$$
h(f)=-\gamma e^{-\frac{f^{2}}{2 \sigma^{2}}}
$$

where $\gamma=128000 / F_{s}, \sigma=7 \cdot\left(3200 / F_{s}\right)^{3}$ and $F_{s}$ is sampling frequency. It should be noticed that the weighting function $h$ is obtained in $\mathrm{dB}$ scale.

As initial points to track the target's body motion, we take $10 \%$ of samples with the highest amplitude from the spectrogram. Then, an unsupervised clusterings is applied to reduce the number of initial points within the neighbour area. Then for each remain initial point, the maximum is tracked within local window with first increasing and then decreasing time index. In such a way we obtain a number of possible tracks. Then the track corresponding to the maximum accumulated energy is selected as the target's body track $V(t)$.

The tracked velocity $V(t)$ is approximated by a polynomial of order 1 to reduce the number of outliers. In such a way the track is assumed to be a simple line. The micro-Doppler signature is shifted then: $\hat{U}(f, t)=U(f+\hat{V}(t), t)$. The last procedure is done by linear interpolation.

Finally, the micro-Doppler signature is ready for feature extraction.

\section{E. Feature extraction}

The features are based on extraction of bases of the microDoppler signature. These bases are orthogonal to each other and contain essential information about the rotating parts of the target. After the alignment we assume that the spectrogram can be viewed as a low rank matrix.

First we need to compute the correlation between different frequency components. Correlation matrix is computed as:

$$
\Psi\left(f_{1}, f_{2}\right)=\sum_{t=1}^{Q} \hat{U}\left(f_{1}, t\right) \hat{U}\left(f_{2}, t\right)
$$

Second step is to estimate eigenpairs $\left\{v_{r}, \lambda_{r}\right\}$, where $v_{r}$ is $r$-th eigenvector and $\lambda_{r}$ corresponds $r$-th eigenvalue, such that $\lambda_{1}>\lambda_{2}>\ldots>\lambda_{r}$. Each eigenpair satisfies the following equality:

$$
\sum_{f_{2}=1}^{M} \Psi\left(f_{1}, f_{2}\right) v_{r}\left(f_{2}\right)=\lambda_{r} v_{r}\left(f_{1}\right)
$$

Eigenvectors are orthogonal and unique forming the basis functions of the signal's spectrum. Eigendecomposition of $\Psi$ or Singular Value Decomposition of $\hat{U}$ can be used for estimation of eigenpairs. The steps (7) and (8) are similar 
to Principal Component Analysis (PCA) with only small difference. For PCA the data must be mean centred before calculating the correlation matrix. In our case the first (and the most important) eigenvector corresponds to the mean vector of the spectrogram.

Next, the Fourier transform of the eigenvectors is computed to obtain features with strong "energy compaction" property, i.e. the features where most of the signal information is concentrated. Typically, the signal information is contained in just a few low-frequency components. Due to this property we can calculate only the $I$ first (low-frequency) coefficients to represent the data:

$$
y_{r}=\bigcup_{i=1}^{I}\left\{\left|\sum_{f=1}^{M} v_{r}(f) e^{-j 2 \pi f l / M}\right|\right\} .
$$

Finally, the feature set is obtained by combining the first five transformed eigenvectors and five eigenvalues:

$$
F=\bigcup_{r=1}^{5}\left\{y_{r}, \lambda_{r}\right\}
$$

\section{ClassificAtion RESUlts}

The proposed ATR system is evaluated on real radar measurements. The radar data have been collected with a continuous-wave radar operating in X-band at radio frequency of $9.5 \mathrm{GHz}$. These radar measurements have been performed within the framework of D-RACE, the Dutch Radar Centre of Expertise, a strategic alliance of Thales Nederland B.V. and TNO. The measured data have been made available for the present study.

The UAVs considered for classification by the proposed technique are listed with their characteristics in Table I. Different types of the UAVs are considered: two planes, a quadrocopter and three helicopters. For comparison, stationary rotating rotors with different number of blades as well as diverse types of birds are considered for classification. At least 30 seconds were recorded of each class. The data were recorded at open space with the distance to target less than $30 \mathrm{~m}$. The aspect angle, distance and velocity were varying as within the real situation.

The sampling frequency of the ADC is set to $32 \mathrm{kHz}$. The radar signal is divided onto a number of segments of fixed length, by processing these segments a decision about the class label is made. The length of one segment is set to 0.5 s. The overlapping between segments is $75 \%$. To compute the STFT we use a sliding Hamming window of length $M=128$ samples $=4 \mathrm{~ms}$ with overlapping of $L=90$ corresponding to $0.9 * 4=3.6 \mathrm{~ms}$. Once the features are extracted the radar signal is down-sampled to $3 \mathrm{kHz}$ and features are extracted again. Finally, the features extracted for the two different sample rates are concatenated to one feature vector. The number of Fourier coefficients in (9) is set to $I=20$.

Examples of micro-Doppler signatures of the considered classes are illustrated in Fig. 1. The classes can be separated
TABLE I

UAVS USED FOR EXPERIMENTAL VERIFICATION OF THE PROPOSED ATR SYSTEM. TYPE 'P' IS FOR PLANE, 'H' IS FOR HELICOPTER, 'Q' FOR QUADROCOPTER, 'B' FOR BIRDS, 'S' FOR STATIONARY ROTORS.

\begin{tabular}{c|c|c|c|c} 
Class & Name & Type & $\#$ of rotors & Rotor, mm \\
\hline \hline 1 & YAK54 vliegtuig & $\mathrm{P}$ & 1 & 100 \\
\hline 2 & EasyStar El-Sailor & $\mathrm{P}$ & 1 & 108 \\
\hline 3 & Birds & $\mathrm{B}$ & - & - \\
\hline 4 & Parrot AR.Drone & $\mathrm{Q}$ & 4 & 200 \\
\hline 5 & 1 rotor & $\mathrm{S}$ & 1 & 203 \\
\hline 6 & 2 rotor & $\mathrm{S}$ & 2 & 203 \\
\hline 7 & 3 rotor & $\mathrm{S}$ & 3 & 203 \\
\hline 8 & 4 rotor & $\mathrm{S}$ & 4 & 203 \\
\hline 9 & Mikado Logo 700 & $\mathrm{H}$ & 2 (main, tail) & 750 (main) \\
\hline 10 & Mikado Logo 400 & $\mathrm{H}$ & 2 (main, tail) & 1040 (main) \\
\hline 11 & Align T-REX 450 & $\mathrm{H}$ & 2 (main, tail) & 715 (main)
\end{tabular}

TABLE II

PROBABILITY OF CORRECT CLASSIFICATION OBTAINED FOR 11 CLASS PROBLEM USING 10-FOLD CROSS VALIDATION.

\begin{tabular}{c|c|c} 
SVM linear & SVM nonlinear & Naive Bayes \\
\hline $94.91 \%$ & $95.39 \%$ & $93.6 \%$
\end{tabular}

even visually. The features we extract are based on eigenvectors of the signature and the first two of them are shown in Fig. 1.

To classify the type of UAV we will use the machine learning approach. All available segments are divided into training and testing sets without overlapping. The decision about class membership is made by a predefined system of rules. The process of defining the system of rules is called learning; it is performed on the training set. The number of correctly classified segments determines the probability of correct classification as ratio to the total number of segments from the testing set. The $\mathrm{K}=10$ cross-validation technique is applied to obtain robust classification rates.

Computed probabilities of correct classification for different classifiers are shown in Table II. The best results are obtained for nonlinear SVM, such $95 \%$ of data are classified correctly. We can notice that all of the classifiers provide similar classification results of the order of $95 \%$ showing the robustness of the extracted features to type of classifier.

Confusion matrix for linear SVM with applied 10-fold cross-validation is shown in Table III to estimate the intra class distribution of classification rates. It can be seen that all targets, except classes 1,2 and 7,8, are classified with probability of correct classification higher than $97 \%$. The first two classes have more errors due to the fact that blades are small and their micro-Doppler signatures are similar. The results for classes 5 to 8 show the capability to distinguish different number of rotors with errors at level of $19 \%$ for distinguishing three from four rotors.

To show the flexibility of the proposed ATR scheme to classify different types of the UAVs, the following classes were removed from the training set: $1,5,6,7,9,10$. In this 

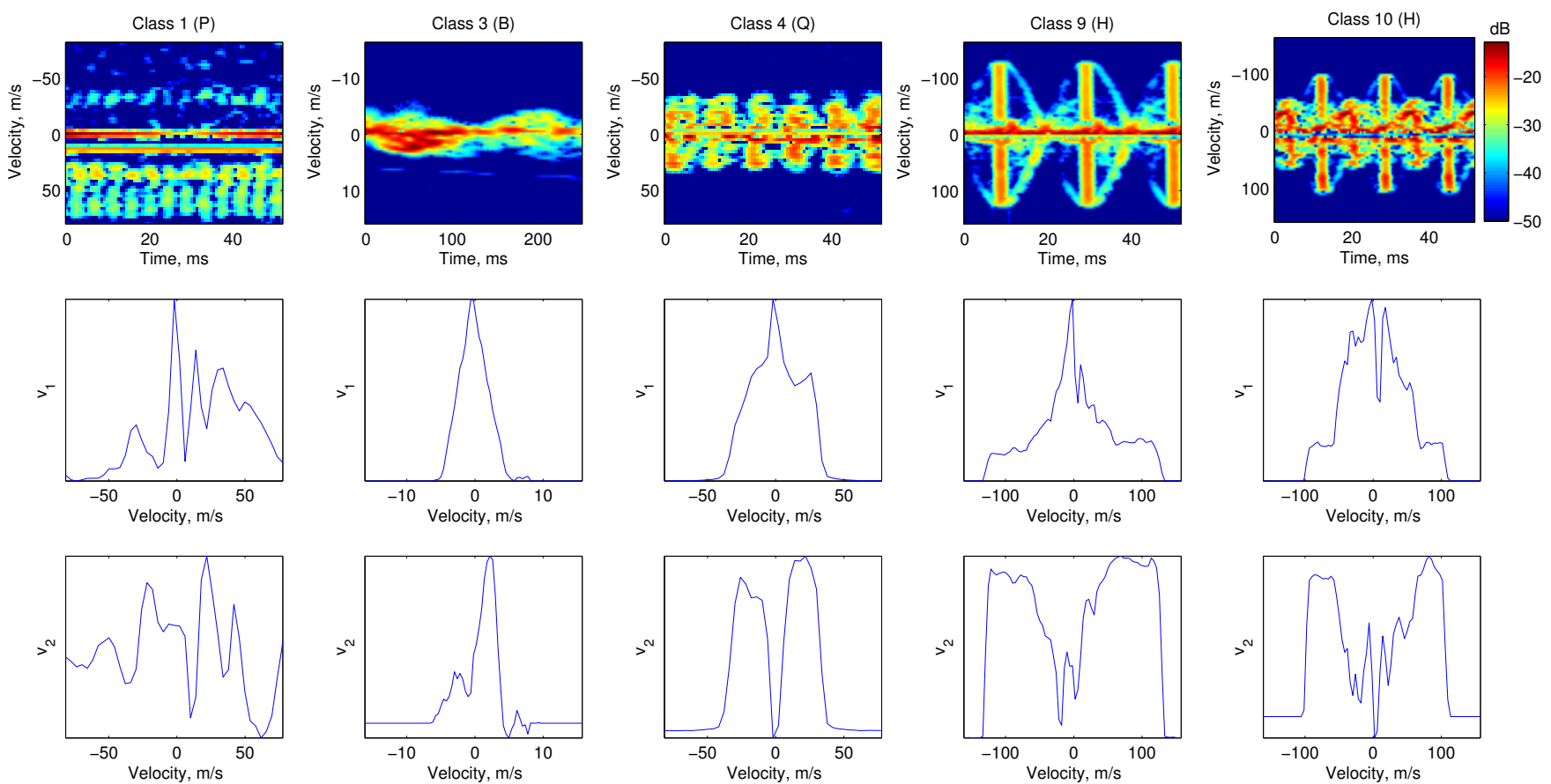

Fig. 1. Examples of filtered and aligned micro-Doppler signatures of 5 classes (first row); Eigenvectors corresponding to the largest eigenvalue (second row) and the second largest eigenvalue (third row) of $\Psi$ for corresponding signatures. These eigenvectors are basis functions of the micro-Doppler signatures.

TABLE III

CONFUSION MATRIX (IN PERCENTAGES) FOR LINEAR SVM CLASSIFIER

\begin{tabular}{c||c|c|c|c|c|c|c|c|c|c|c} 
class & 1 & 2 & 3 & 4 & 5 & 6 & 7 & 8 & 9 & 10 & 11 \\
\hline \hline 1 & $\mathbf{8 6}$ & 13 & 0 & 1 & 0 & 0 & 0 & 0 & 0 & 0 & 0 \\
\hline 2 & 11 & $\mathbf{8 9}$ & 0 & 0 & 0 & 0 & 0 & 0 & 0 & 0 & 0 \\
\hline 3 & 1 & 0 & $\mathbf{9 7}$ & 2 & 0 & 0 & 0 & 0 & 0 & 0 & 0 \\
\hline 4 & 0 & 0 & 1 & $\mathbf{9 9}$ & 0 & 0 & 0 & 0 & 0 & 0 & 0 \\
\hline 5 & 0 & 0 & 0 & 0 & $\mathbf{1 0 0}$ & 0 & 0 & 0 & 0 & 0 & 0 \\
\hline 6 & 0 & 0 & 0 & 0 & 2 & $\mathbf{9 8}$ & 0 & 0 & 0 & 0 & 0 \\
\hline 7 & 0 & 0 & 0 & 0 & 0 & 0 & $\mathbf{8 1}$ & 19 & 0 & 0 & 0 \\
\hline 8 & 0 & 0 & 0 & 0 & 0 & 0 & 6 & $\mathbf{9 4}$ & 0 & 0 & 0 \\
\hline 9 & 0 & 0 & 0 & 0 & 0 & 0 & 0 & 0 & $\mathbf{1 0 0}$ & 0 & 0 \\
\hline 10 & 0 & 0 & 0 & 0 & 0 & 0 & 0 & 0 & 0 & $\mathbf{1 0 0}$ & 0 \\
\hline 11 & 0 & 0 & 0 & 0 & 0 & 0 & 0 & 0 & 1 & 0 & $\mathbf{9 9}$
\end{tabular}

way for each type of the UAVs (plane, birds, stationery rotors, quadrocopter and helicopter) only a single class is used for training. The removed classes are then used to test the system. The results of classification by linear SVM classifier are listed in the Table IV. We can claim that proposed ATR scheme is robust to variations inside the class, and therefore with high probability, other UAVs with similar flying concept will be classified correctly.

\section{CONCLUSIONS}

A new automatic target recognition system has been proposed for classification of unmanned aerial targets by their micro-Doppler signatures. The preprocessing steps of the
TABLE IV

CLASSIFICATION OF UAVS HIDDEN FROM TRAINING PROCEDURE

\begin{tabular}{c||c|c|c|c|c} 
Class & $\mathrm{P}$ & $\mathrm{B}$ & $\mathrm{Q}$ & $\mathrm{S}$ & $\mathrm{H}$ \\
\hline 1 & $\mathbf{8 7}$ & 8 & 5 & 0 & 0 \\
\hline 5 & 0 & 0 & 0 & $\mathbf{1 0 0}$ & 0 \\
\hline 6 & 0 & 0 & 0 & $\mathbf{1 0 0}$ & 0 \\
\hline 7 & 0 & 0 & 0 & $\mathbf{1 0 0}$ & 0 \\
\hline 9 & 0 & 0 & 0 & 0 & $\mathbf{1 0 0}$ \\
\hline 10 & 0 & 0 & 0 & 0 & $\mathbf{1 0 0}$
\end{tabular}

signature such as filtering and Doppler alignment have been discussed. New, robust features for target classification based on extraction of bases of the micro-Doppler signature have been proposed. The probability of correct classification of the order of $95 \%$ has been achieved. It has been shown that the type of the UAV can be determined correctly even if it was hidden from the training set.

\section{REFERENCES}

[1] V. Chen, The Micro-Doppler Effect in Radar. London: Artech House, 2011.

[2] J. J. M de Wit, R. I. A. Harmanny, and G. Premel-Cabic, "Micro-Doppler analysis of small uavs," in Radar Conference (EuRAD), 2012 European, oct. 2012 , pp. $210-213$.

[3] C. Clemente, A. Balleri, K. Woodbridge, and J. Soraghan, "Developments in target micro-Doppler signatures analysis: radar imaging, ultrasound and through-the-wall radar," EURASIP Journal on Advances in Signal Processing, vol. 2013, no. 1, p. 47, 2013.

[4] S. Vaseghi, Advanced digital signal processing and noise reduction. John Wiley, 2000. 